\title{
Heating and cooling of the neutral ISM in the NGC 4736 circumnuclear ring
}

T. P. R. van der Laan ${ }^{1,2}$, L. Armus ${ }^{2}$, P. Beirao ${ }^{3}$, K. Sandstrom ${ }^{4}$, B. Groves ${ }^{5}$, E. Schinnerer ${ }^{5}$, B. T. Draine ${ }^{6}$, J. D. Smith ${ }^{7}$, M. Galametz ${ }^{8}$, M. Wolfire ${ }^{9}$, K. Croxall ${ }^{10}$, D. Dale ${ }^{11}$, R. Herrera Camus ${ }^{9}$, D. Calzetti ${ }^{12}$, and R. C. Kennicutt, Jr. ${ }^{13}$

${ }^{1}$ Institut de Radioastronomie Millimétrique (IRAM), 300 rue de la Piscine, 38406 St. Martin d'Hères, Grenoble, France e-mail: vanderlaan@iram.fr

2 Spitzer Science Center, California Institute of Technology, MC 314-6, Pasadena, CA 91125, USA

3 Observatoire de Paris, 61 avenue de l'Observatoire, 75014 Paris, France

4 Steward Observatory, University of Arizona, 933 N. Cherry Ave, Tucson, AZ 85721, USA

5 Max-Planck-Institut für Astronomie, Königstuhl 17, 69117 Heidelberg, Germany

6 Department of Astrophysical Sciences, Princeton University, Princeton, NJ 08544, USA

7 Department of Physics and Astronomy, University of Toledo, Toledo, OH 43606, USA

${ }^{8}$ European Southern Observatory, Karl-Schwarzchild-Str. 2, 85748 Garching-bei-München, Germany

9 Department of Astronomy, University of Maryland, College Park, MD 20742, USA

10 Department of Astronomy, The Ohio State University, 140 West 18th Avenue, Columbus, OH 43210, USA

11 Department of Physics \& Astronomy, University of Wyoming, Laramie, WY 82071, USA

12 Department of Astronomy, University of Massachusetts, Amherst, MA 01003, USA

13 Institute of Astronomy, University of Cambridge, Madingley Road, Cambridge CB3 0HA, UK

Received 25 November 2014 / Accepted 21 January 2015

\section{ABSTRACT}

\begin{abstract}
The manner in which gas accretes and orbits within circumnuclear rings has direct implications for the star formation process. In particular, gas may be compressed and shocked at the inflow points, resulting in bursts of star formation at these locations. Afterwards the gas and young stars move together through the ring. In addition, star formation may occur throughout the ring, if and when the gas reaches sufficient density to collapse under gravity. These two scenarios for star formation in rings are often referred to as the "pearlson-a-string" and "popcorn" paradigms. In this paper, we use new Herschel/PACS observations, obtained as part of the KINGFISH open time key program, along with archival Spitzer and ground-based observations from the SINGS Legacy project, to investigate the heating and cooling of the interstellar medium in the nearby star-forming ring galaxy, NGC 4736. By comparing spatially resolved estimates of the stellar far-ultraviolet flux available for heating, with the gas and dust cooling derived from the far-infrared continuum and line emission, we show that while star formation is indeed dominant at the inflow points in NGC 4736, additional star formation is needed to balance the gas heating and cooling throughout the ring. This additional component most likely arises from the general increase in gas density in the ring over its lifetime. Our data provide strong evidence, therefore, for a combination of the two paradigms for star formation in the ring in NGC 4736.
\end{abstract}

Key words. galaxies: individual: NGC 4736 - galaxies: ISM - galaxies: star formation

\section{Introduction}

Circumnuclear star formation rings are present in approximately $20 \%$ of nearby disk galaxies (Comerón et al. 2010). Early theories suggested that the radius at which the rings form was the inner Lindblad resonance (ILR) radius, which is set by an asymmetric component in the stellar disk such as a large scale bar. However, further modeling of galaxy gravitational potentials has shown that circumnuclear ring formation is linked more particularly to the presence of a sufficient number of $x_{2}$ orbits (Regan $\&$ Teuben 2003). Such orbits are (near-)circular, in contrast to the elongated $x_{1}$ orbits at larger radii, which make up the large scale bar (Contopoulos \& Papayannopoulos 1980). The radii of $x_{2}$ orbits are close to the ILR radius.

As gas and dust lose angular momentum, they move inward. At the overlap between $x_{1}$ to $x_{2}$ orbits, gas and dust will settle on the lower energy $x_{2}$ orbits as a result of collisions, forming a circumnuclear ring. The discrete locations where this transition happens are generally called "inflow points". Such inflow points are most obvious in ring systems with large scale stellar bars, where the connection between the spiral shocks of the bar and the circumnuclear ring can often be clearly seen (for example
NGC 6951, Van der Laan et al. 2011). However, circumnuclear rings also exist in galaxies classified as non-barred (see, e.g., Grouchy et al. 2010, for a discussion on the difference, or lack thereof, between barred and non-barred systems in this context). Any gravitational asymmetry in the disk may suffice, as long as enough $x_{2}$ orbits are present in the gravitational potential.

The gas and dust can experience shocks as they transition from $x_{1}$ to $x_{2}$ orbits at the inflow points. However, after the gas and dust settles on the $x_{2}$ orbits of the ring, the accreted ISM experiences very little large-scale friction. With that in mind, it is not too surprising that it has been found in multiple galaxies that most (if not all) star formation in circumnuclear rings takes place at the inflow points (Ryder et al. 2001; Allard et al. 2005; Böker et al. 2008; Mazzuca et al. 2008; Van der Laan et al. 2013a,b; Falcón-Barroso et al. 2014). Consequently, it is thought that, owing to the absence of large scale friction or temporal variations in gravitational potential, stars and gas primarily move through the circumnuclear ring together. The stellar age gradient regularly seen stretching out from each inflow point is often referred to as "pearls-on-a-string", first coined by Böker et al. (2008). An alternative theory (Elmegreen 1994) suggests that the inflow points play no special role in star formation, and that a build up 
of gas will trigger a burst of star formation after a critical limit has been reached anywhere in a ring. This is often referred to as the "popcorn" theory of star formation in rings.

Recent star formation is often tracked by observing hydrogen recombination lines such as $\mathrm{H} \alpha$ or $\operatorname{Br} \gamma$. The equivalent width of the Balmer lines is, for example, dependent on stellar age from 4 Myr (e.g. Leitherer \& Heckman 1995; Bresolin \& Kennicutt 1997; González Delgado et al. 1999). Alternatively, emission lines from other atoms and molecules stimulated by stellar radiation, such as warm $\mathrm{H}_{2}$, HeI, and [FeII], in the optical and near-infrared (NIR) can be used. With a combination of these lines it is also possible to observe potential age gradients, as demonstrated for the circumnuclear ring in NGC 613 (Böker et al. 2008; Falcón-Barroso et al. 2014). However, strong extinction can make observations in the optical and NIR range difficult, especially at the inflow points where dust and gas build up, potentially limiting the effectiveness of these methods.

When there are no large-scale mechanical stresses, stellar radiation is most likely to be the prevalent form of heating for the neutral gas in the ring (excluding contributions of CR or X-ray ionization). Thus, an alternative way of tracking recent star formation is to study the heating and cooling of the neutral interstellar medium (ISM) via strong emission lines of the neutral gas in the far-infrared (FIR). In the presence of star formation, the heating of the neutral ISM is dominated by photo-electric heating (Bakes \& Tielens 1994; Weingartner \& Draine 2001; Helou et al. 2001). Dust grains and PAHs eject electrons as the result of the absorption of far-ultraviolet (FUV; $6 \mathrm{eV}<h v<13.6 \mathrm{eV}$ ) photons from young $\mathrm{O}$ and $\mathrm{B}$ type stars. These electrons in turn interact with the gas, heating atoms and molecules. The cooling times for dust and gas are much shorter than the Myr astronomical timescales of interest here. Thus, gas heating and cooling, via photo-electric heating, may be considered in balance at all times, and the observed cooling flux can be used as a proxy for the heating.

To test whether the heating of the neutral ISM in the ring is indeed dominated by stellar radiative heating from stars formed at the inflow points, we investigate the case of the circumnuclear ring in NGC 4736, a nearby $\left(D=4.66 \mathrm{Mpc}, i \sim 35^{\circ}\right.$, $\mathrm{PA} \sim 300^{\circ}$ ) SAab spiral galaxy. The ring in NGC 4736 has a radius of $40^{\prime \prime}(0.9 \mathrm{kpc})$. Based on a previously observed HI velocity field (Walter et al. 2008) and assuming that the spiral arms are concave, the north side of the disk is the near side, and the south the far side, with a clockwise rotation. We have combined available data to form a large multiwavelength dataset, tracing the gas and the recent star formation, including Herschel/PACS data from the KINGFISH (Key Insights on Nearby Galaxies: A Far-Infrared Survey with Herschel) open time key program (Kennicutt et al. 2011). The PACS observations enable us to probe the main neutral gas cooling lines of [CII] at $158 \mu \mathrm{m}$ and [OI] at $63 \mu \mathrm{m}$, as well as the FIR dust continuum. We also include data obtained with the Spitzer Space Telescope as part of the SINGS (Spitzer Infrared Nearby Galaxies Survey) Legacy program (Kennicutt et al. 2003), to probe the mid-infrared wavelength range, which includes emission from large dust grains, PAH's, stars, and molecular and atomic gas.

In Sect. 2 we present the data. In Sect. 3 the atomic and molecular gas mass surface densities, and subsequent dust mass surface density, are computed. In Sect. 4 a census is made of the star formation rate density throughout the ring. In Sect. 5 the contribution of the neutral ISM to the observed flux is determined. We then compare the dust and gas cooling with the stellar heating in Sect. 6. The implications are discussed in Sect. 7, with a summary in Sect. 8.

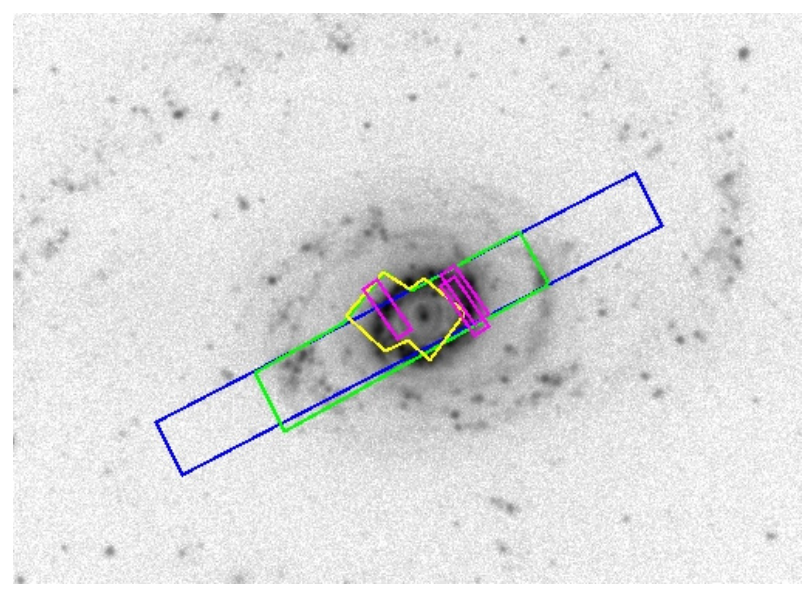

Fig. 1. Spatial coverage of the spectral data sets presented in this study, overlaid on the GALEX FUV image of NGC 4736. The circumnuclear ring at a radius of $r \sim 40^{\prime \prime}(0.9 \mathrm{kpc})$ stands out clearly in the GALEX image. The different regions mapped spectroscopically, and indicated here in color, are: Spitzer/IRS long-low $(14-38 \mu \mathrm{m})$ spectra (dark blue contours), Herschel/PACS [CII] and [OI] emission line maps (green contours), Herschel/PACS [NII]122 $\mu \mathrm{m}$, [NII]205 $\mu \mathrm{m}$ emission line maps (yellow contours), and Spitzer/IRS short-low (5-14 $\mu \mathrm{m})$ spectra (purple contours). In this image, north is up and east is to the left, and the total size is approximately 7.3 arc minutes on a side.

\section{Data sets}

\subsection{Herschel/PACS}

Both spectra and images were obtained for NGC 4736 with the Herschel/PACS instruments as part of the KINGFISH open time key program. The PACS spectroscopic instrument is an integral field unit (IFU) with $5 \times 5,9$ '. 4 pixels (Poglitsch et al. 2010). This gives an instantaneous field-of-view (FoV) of $47^{\prime \prime} \times 47^{\prime \prime}$. The PACS beam FWHM is 5 ". 2 in the wavelength range $60-85 \mu \mathrm{m}$, and $12^{\prime \prime}$ at $130-210 \mu \mathrm{m}$. For the spectra several targeted pointings were made for the various lines. The [NII]122 $\mu \mathrm{m}$ and [NII] $205 \mu \mathrm{m}$ lines were observed with 2 adjacent pointings, one focussed on the nucleus and one on the circumnuclear ring (see yellow contours in Fig. 1). The [CII] $158 \mu \mathrm{m}$ and [OI]63 $\mu \mathrm{m}$ lines were observed in a strip 200" long (green contours), with an orientation equal to previous Spitzer/IRS data (blue contours), and one adjacent pointing on the circumnuclear ring (equal to the [NII] off-nucleus pointing). The FIR continuum was probed at $70 \mu \mathrm{m}, 100 \mu \mathrm{m}$, and $160 \mu \mathrm{m}$ with the PACS photometer over the full extent of the galaxy.

The PACS observations were reduced consistently for the complete KINGFISH sample using the Scanamorphus package (Roussel 2013). The KINGFISH observations are described in Dale et al. (2012) and Croxall et al. (2012). In all cases, the images were rescaled to a pixel scale of $4^{\prime \prime}$. The spectra zerothmoment maps were convolved to the PACS $160 \mu \mathrm{m}$ resolution of $12^{\prime \prime}$, using a pixel scale of $4^{\prime \prime}$, since the PACS 160 beam is essentially the same as the [CII] beam.

The $[\mathrm{CII}]$ and $[\mathrm{OI}]$ intensity maps, as well as the totalinfrared (TIR) continuum map are shown in Fig. 2. The TIR continuum is constructed from a combination of four individual Spitzer and Herschel images, at $8 \mu \mathrm{m}, 24 \mu \mathrm{m}, 70 \mu \mathrm{m}$, and $160 \mu \mathrm{m}$, following the equation in Draine \& Li (2007),

$\mathrm{TIR}=\left(0.95\left\langle v S_{v}\right\rangle_{8}+1.15\left\langle v S_{v}\right\rangle_{24}+\left\langle v S_{v}\right\rangle_{70}+\left\langle v S_{v}\right\rangle_{160}\right)$.

Even though the FoV of the spectra clips the northern and southern part of the circumnuclear ring, the [CII] and [OI] emission 

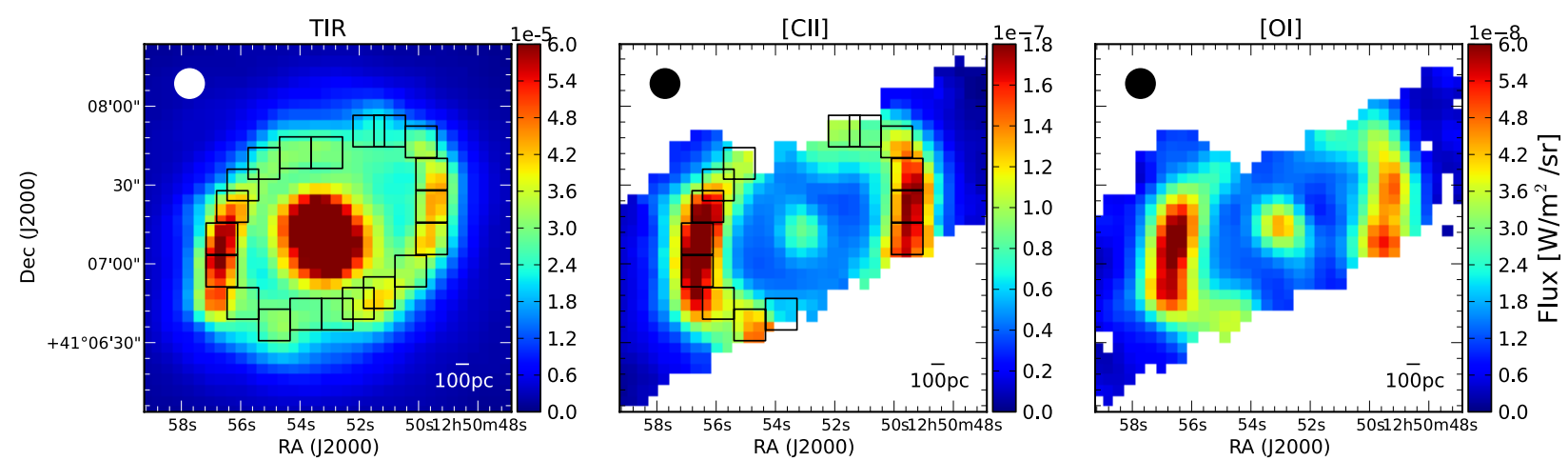

Fig. 2. Distribution of the total infrared (TIR) continuum emission (left), [CII] $158 \mu \mathrm{m}$ line emission (middle), and [OI] $63 \mu \mathrm{m}$ line emission (right) in the circumnuclear ring of NGC 4736. The 12" apertures used to extract fluxes are indicated in the TIR and [CII] panels. The spatial scale is given in the bottom right corner, and the PACS $160 \mu \mathrm{m}$ beam of $\sim 12^{\prime \prime}$ is indicated in the top left corner of each panel.

Table 1. [CII], [OI], and TIR surface brightnesses in defined apertures.

\begin{tabular}{rccccccccc}
\hline \hline$\#$ & $\begin{array}{c}\text { pos } x \\
{[\mathrm{pix}]}\end{array}$ & $\begin{array}{c}\text { pos } y \\
{[\mathrm{pix}]}\end{array}$ & $\begin{array}{c}\text { Angle } \\
{[\mathrm{deg}]}\end{array}$ & $\begin{array}{c}{[\mathrm{CII}]} \\
\mathrm{W} / \mathrm{m}^{2} / \mathrm{sr}\end{array}$ & $\begin{array}{c}{[\mathrm{CII}]_{\mathrm{unc}}} \\
\text { rel. }\end{array}$ & $\begin{array}{c}{[\mathrm{OI}]} \\
\mathrm{W} / \mathrm{m}^{2} / \mathrm{sr}\end{array}$ & $\begin{array}{c}{[\mathrm{OI}]_{\mathrm{unc}}} \\
\text { rel. }\end{array}$ & $\begin{array}{c}\mathrm{TIR} \\
\mathrm{W} / \mathrm{m}^{2} / \mathrm{sr}\end{array}$ & $\begin{array}{c}\mathrm{TIR}_{\text {unc }} \\
\text { rel. }\end{array}$ \\
\hline 1 & 149 & 144 & 15 & - & - & - & - & $1.29 \mathrm{e}-4$ & 0.09 \\
2 & 146 & 143 & 29 & $1.12 \mathrm{e}-6$ & 0.01 & $2.43 \mathrm{e}-7$ & 0.12 & $2.64 \mathrm{e}-4$ & 0.07 \\
3 & 143 & 145 & 49 & $1.13 \mathrm{e}-6$ & 0.01 & $3.33 \mathrm{e}-7$ & 0.06 & $2.84 \mathrm{e}-4$ & 0.04 \\
4 & 141 & 148 & 68 & $1.62 \mathrm{e}-6$ & 0.01 & $4.91 \mathrm{e}-7$ & 0.04 & $4.54 \mathrm{e}-4$ & 0.04 \\
5 & 141 & 151 & 85 & $1.75 \mathrm{e}-6$ & 0.01 & $5.40 \mathrm{e}-7$ & 0.03 & $4.88 \mathrm{e}-4$ & 0.03 \\
6 & 142 & 154 & 102 & $1.60 \mathrm{e}-6$ & 0.01 & $4.24 \mathrm{e}-7$ & 0.05 & $3.98 \mathrm{e}-4$ & 0.03 \\
7 & 143 & 156 & 117 & $1.11 \mathrm{e}-6$ & 0.01 & $2.72 \mathrm{e}-7$ & 0.09 & $2.94 \mathrm{e}-4$ & 0.03 \\
8 & 145 & 158 & 135 & $8.87 \mathrm{e}-7$ & 0.02 & $2.00 \mathrm{e}-7$ & 0.23 & $2.56 \mathrm{e}-4$ & 0.04 \\
9 & 148 & 159 & 157 & - & - & - & - & $2.70 \mathrm{e}-4$ & 0.03 \\
10 & 151 & 159 & 180 & - & - & - & - & $2.56 \mathrm{e}-4$ & 0.03 \\
11 & 155 & 161 & 205 & $8.50 \mathrm{e}-7$ & 0.02 & $1.31 \mathrm{e}-7$ & 0.19 & $2.20 \mathrm{e}-4$ & 0.03 \\
12 & 157 & 161 & 213 & $7.83 \mathrm{e}-7$ & 0.02 & $1.69 \mathrm{e}-7$ & 0.11 & $2.12 \mathrm{e}-4$ & 0.03 \\
13 & 160 & 160 & 229 & $1.05 \mathrm{e}-6$ & 0.01 & $2.94 \mathrm{e}-7$ & 0.06 & $2.66 \mathrm{e}-4$ & 0.03 \\
14 & 161 & 157 & 243 & $1.34 \mathrm{e}-6$ & 0.01 & $3.70 \mathrm{e}-7$ & 0.05 & $3.28 \mathrm{e}-4$ & 0.03 \\
15 & 161 & 154 & 259 & $1.44 \mathrm{e}-6$ & 0.01 & $3.82 \mathrm{e}-7$ & 0.05 & $3.54 \mathrm{e}-4$ & 0.03 \\
16 & 161 & 151 & 275 & $1.40 \mathrm{e}-6$ & 0.01 & $4.34 \mathrm{e}-7$ & 0.08 & $3.38 \mathrm{e}-4$ & 0.03 \\
17 & 159 & 148 & 297 & - & - & - & - & $2.88 \mathrm{e}-4$ & 0.05 \\
18 & 156 & 146 & 320 & - & - & - & - & $3.42 \mathrm{e}-4$ & 0.10 \\
19 & 154 & 145 & 337 & - & - & - & - & $3.14 \mathrm{e}-4$ & 0.12 \\
20 & 152 & 144 & 353 & - & - & - & - & $2.56 \mathrm{e}-4$ & 0.12 \\
\hline
\end{tabular}

Notes. $[\mathrm{CII}]$ and $[\mathrm{OI}]$ emission line fluxes, TIR continuum flux, and their relative uncertainties in $3 \times 3$ pixel $\left(12^{\prime \prime} \times 12^{\prime \prime}\right)$ apertures. Central positions (in TIR map pixels) of the apertures are given in the 2 nd and 3rd columns. The order of the apertures (angles) here is clockwise (subsequently following the direction of gas motion), starting from due south. For reference, pixel $(151,152)$ corresponds to the galaxy center (RA $12 \mathrm{~h} 50 \mathrm{~m} 53.18 \mathrm{~s}$, Dec $41 \mathrm{~d} 07 \mathrm{~m} 12.0 \mathrm{~s})$.

is clearly concentrated there. The TIR continuum also has elevated emission at those locations, but is mostly dominated by the nucleus.

Integrated fluxes are extracted by covering the ring with $3 \times 3$ pixel $\left(12^{\prime \prime} \times 12^{\prime \prime}\right)$ apertures. In this manner we measured the $[\mathrm{CII}]$ and $[\mathrm{OI}]$ emission line and continuum fluxes at 13 individual points, seven on the east side, and six on the west side of the ring. For the TIR continuum an additional six apertures are positioned on the ring outside the spectroscopic FoV (see Fig. 2). This east (west) side corresponds to the blue (red) shifted side of the galaxy. The positions of, and fluxes in, these $12^{\prime \prime}$ apertures are given in Table 1.

\subsection{Spitzer}

As part of the SINGS survey NGC 4736 was observed with Spitzer/IRS using the "short-low" and "long-low" spectral modules in spectral mapping mode. The circumnuclear ring is incompletely covered by the "long-low" footprint (see again Fig. 1, blue contour), and partially by the three off-nuclear "short-low" pointings (purple contours). The "short-low" (SL) module has a pixel scale of 1". 85 and a resolving power of 60-120, decreasing with increasing wavelength. The observations have a FoV of 3'. 6 by 27 " and cover the wavelength range 5-14 $\mu \mathrm{m}$. The "longlow" (LL) module has a pixel scale of 5". 1, a resolving power of approximately 60-120, again decreasing with wavelength, a FoV of $10 . ' 6$ by $84^{\prime \prime}$, and covers the wavelength range $14-28 \mu \mathrm{m}$. The reduced and fully calibrated data cubes are available on the SINGS Spitzer and Ancillary Data website ${ }^{1}$.

For this work we extracted the [SIII] emission line flux at $18.7 \mu \mathrm{m}$ and $33.5 \mu \mathrm{m}$ by fitting the lines and the underlying warm dust continuum with PAHFIT (Smith et al. 2007) on the LL data cube alone. The [SIII] emission lines are not blended, and the dust emission is a smoothly varying composition of black-body curves of different temperatures. Consequently, the separation of line and continuum emission at these wavelengths

1 http://irsa.ipac.caltech.edu/data/SPITZER/SINGS/ 

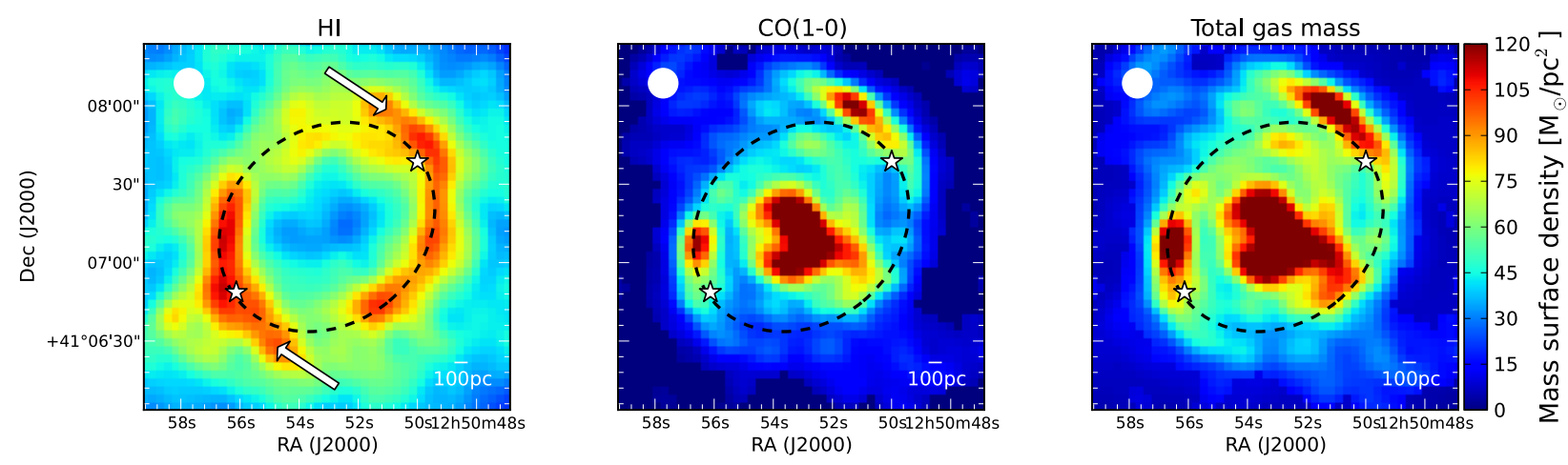

Fig. 3. Left and middle: mass surface density maps of atomic (HI) and molecular $(\mathrm{CO}(1-0))$ gas, at the PACS $160 \mu \mathrm{m}\left(\sim 12^{\prime \prime}\right)$ resolution. Right: total gas mass surface density map, based on HI and CO maps, with a $26 \%$ correction for helium. The radius of the circumnuclear ring and the locations of the inflow points are indicated with a dashed circle and stars, respectively. Clockwise gas flow is indicated with the white arrows.

with PAHFIT is straight-forward. A check at the positions with both SL and LL data showed that the exclusion of the SL data cubes had no detrimental effect on the fitting of the longwavelength dust continuum. Since the IRS data are not combined with the other data sets - they will only be used in Sect. 5 - and have a limited FoV, no effort was made to convolve the cubes to the PACS160 resolution.

Spitzer/IRAC imaging at $8 \mu \mathrm{m}$ and $24 \mu \mathrm{m}$ are also presented, and were convolved to the PACS160 resolution using kernels from Aniano et al. (2011).

\subsection{Ancillary data}

The THINGS survey ${ }^{2}$ (Walter et al. 2008) observed the HI distribution of NGC 4736 with the VLA between March 2003 and March 2004 in the BCD configurations. The HI intensity maps (natural and robust weighting) are publicly available from the THINGS website. The beam size of the observations is, depending on the chosen weighting, 10.'22 ×9!'07 (natural), or $5{ }^{\prime \prime} 96 \times 5$ '. 55 (robust).

The BIMA SONG survey (Helfer et al. 2003) observed the CO(1-0) distribution in NGC 4736 in October 1997. The total FoV of these data are $\sim 190^{\prime \prime}(4.3 \mathrm{kpc})$. The data have a resolution of $6^{\prime \prime}$ and are short spacing corrected. The resulting data cube and moment maps are publicly available from NED.

NGC 4736 was observed with GALEX in both the nearultraviolet (NUV) and FUV bands between April and June, 2004. The observations are publicly available through the MAST GALEX public access site ${ }^{3}$. The FoV is $1.2^{\circ}$, with a FWHM angular resolution of 4.'0 (NUV) and 5.'6 (FUV).

An $\mathrm{H} \alpha$ map was obtained from the NASA/IPAC Extraglactic Database (NED), but was originally observed, reduced, and continuum and [NII] subtracted by Knapen et al. (2004). It was obtained with the Isaac Newton $2.5 \mathrm{~m}$ telescope. The angular resolution is $11^{\prime \prime} 4 \times 11^{\prime \prime} 4$ and the pixel scale is 0 ! 3 .

\section{ISM distribution in the ring}

\subsection{Atomic and molecular gas}

Both the $\mathrm{HI}$ and $\mathrm{CO}(1-0)$ intensity distributions, convolved to the PACS $160 \mu \mathrm{m}$ resolution, are shown in the first two panels of Fig. 3. The HI distribution is dominated by two arcs which are co-spatial with the circumnuclear star forming ring (whose

\footnotetext{
2 http://www.mpia-hd.mpg.de/THINGS/Overview.html

3 http://galex.stsci.edu/GR6/
}

radius is approximated with the dashed ellipse, representing an $i \sim 35^{\circ}$ inclined ring). The nucleus is devoid of atomic gas. By comparison, the $\mathrm{CO}(1-0)$ distribution shows emission in a more spiral arm-like pattern. (This is even more apparent at native resolution.) The spiral arms only partly overlap with the circumnuclear ring. Unlike the $\mathrm{HI}$, the $\mathrm{CO}(1-0)$ intensity distribution further shows strong emission from the nucleus.

The "total" gas mass density is computed via these two tracers, by assuming a CO-conversion factor, $\mathrm{X}_{\mathrm{CO}}$, of $7.4 \times 10^{19}\left[\mathrm{~cm}^{-2}\left(\mathrm{~K} \mathrm{~km} \mathrm{~s}^{-1}\right)^{-1}\right]$ (Sandstrom et al. 2013). This value is lower than the Galactic $X_{C O}$ value of $2.0 \times$ $10^{20}\left[\mathrm{~cm}^{-2}\left(\mathrm{~K} \mathrm{~km} \mathrm{~s}^{-1}\right)\right]$ most often assumed for nearby galaxies, but Sandstrom et al. (2013) have shown that $\mathrm{X}_{\mathrm{CO}}$ drops to this value towards the galactic nuclear region of this galaxy (averaged at a resolution of $37^{\prime \prime} .5$ ). Due to the lower $\mathrm{X}_{\mathrm{CO}}$ factor, the molecular gas composes a smaller fraction of the total gas mass. The total gas mass map is still dominated by molecular gas, as can be expected in the molecule rich centers of galaxies, but atomic gas provides about $1 / 3$ of the gas mass in the ring. A $26 \%$ correction for helium and metals has been applied to the total gas map.

Ring inflow points are defined as the position where the gas spiral arm(s) cross onto the ring. In a bi-symmetric potential, e.g. with a stellar bar, the two points should be more or less symmetric about the nucleus. An indication of their position in this galaxy is given in Fig. 3. The total gas mass map shows two, possibly three, regions of high mass surface density at the radius of the ring, in the east, the northwest, and at lower significance to the southwest. The first two are near the inflow points.

\subsection{Dust}

By comparing our gas mass map with a map of the dust mass, we can determine whether or not the dust and gas in this region are well mixed, which is a necessary condition for our upcoming analysis and comparison to models of the star formation in the ring. The dust mass distribution in NGC 4736 is derived using via the Draine \& Li (2007) model, including Spitzer, Herschel/PACS ${ }^{4}$ and SPIRE observations of the source, to constrain the IR dust emission (Aniano et al. 2012). We find that our mass distribution of gas shows a one-on-one morphology correspondence, confirming that dust and gas are qualitatively well mixed. Equally, when we compare our TIR continuum map (Fig. 2) with the dust luminosity found by the model a one-onone morphology correspondence can be observed. Additionally

\footnotetext{
4 The same observations as used in this work.
} 

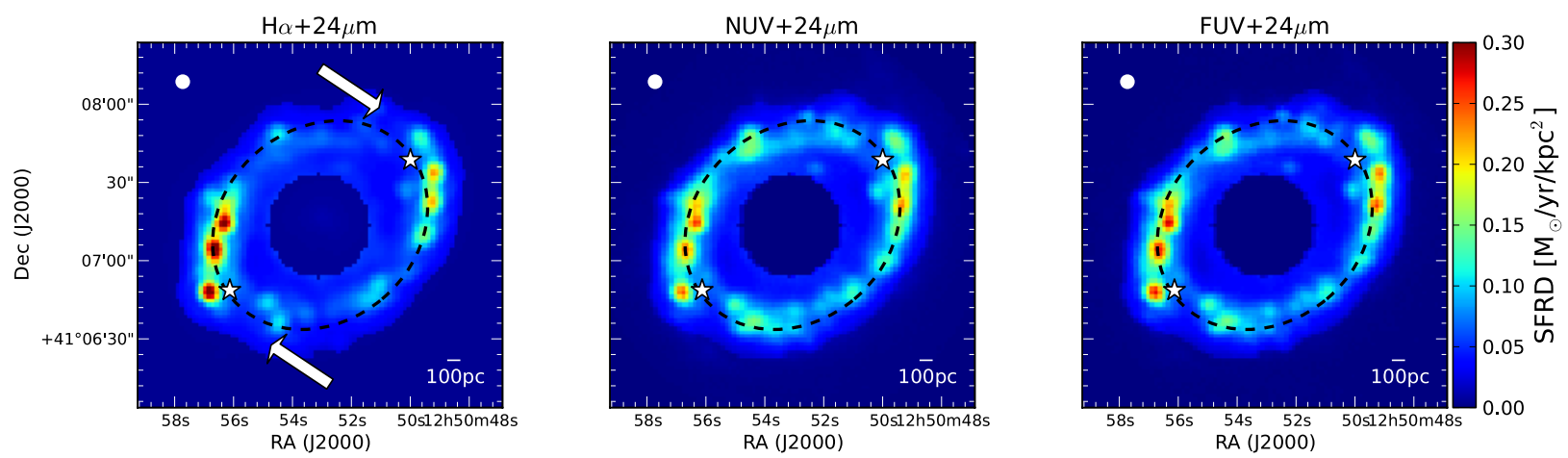

Fig. 4. Star formation rate density maps, based on $\mathrm{H} \alpha+24 \mu \mathrm{m}$ (left), NUV $+24 \mu \mathrm{m}$ (middle), and FUV $+24 \mu \mathrm{m}$ (right), at the Spitzer $24 \mu \mathrm{m}$ $\left(\sim 5^{\prime \prime}\right)$ resolution. The $\mathrm{H} \alpha+24 \mu \mathrm{m}$ derived SFRD values in this image have been multiplied by a factor two for visualization purposes. The galactic nucleus and larger radii have been masked. The radius of the circumnuclear ring and the locations of the inflow points are indicated with a dashed circle and stars, respectively. The Spitzer $24 \mu \mathrm{m}$ beam of $\sim 5^{\prime \prime}$ is indicated in the top left corner of each subpanel.

we find that our total gas mass map also matches quantitatively, i.e., with reasonable conversion values for the dust-to-gas ratio (DGR) we recover the values in the dust map.

\section{Star formation distribution in the ring}

The star formation rate in the circumnuclear ring can be derived from either the $\mathrm{H} \alpha$, NUV, or FUV data sets. To compensate for the stellar light absorbed by dust, all three star formation rate tracers are combined with the Spitzer $24 \mu \mathrm{m}$ image to obtain dust-corrected values.

The star formation rate is computed based on the SFR calibrations given in Murphy et al. (2011), Hao et al. (2011), and Kennicutt \& Evans (2012). The resulting SFR surface density (SFRD) maps are shown in Fig. 4. Differences between the three maps reflect the uncertainties and physical/temporal variations among the SFR tracers. The region for which the SFRD is computed is, in all cases, limited at small radii by a circular mask at $20^{\prime \prime}$ to exclude emission from the nucleus. At the outer edge, a limit has been placed at the minimum of either $70^{\prime \prime}$ radius, or the position where the flux in the tracers falls to a $2 \sigma$ level. The maps shown in Fig. 4 are at the resolution of the $24 \mu \mathrm{m}$ observations, which is $\sim 5^{\prime \prime}$, and have a pixel scale of 1 ".5. SFRD maps at the PACS $160 \mu \mathrm{m}$ resolution were also computed.

In all three panels, the regions of highest star formation rates are found in the southeast and northwest quadrants of the ring. These positions are near/at the ring inflow points, as expected. At the other two quadrants of the ring, the SFRD is lower (by factors of three to six). This could indicate a secondary star formation event of lower-level intensity, or the continued emission from an ageing burst. The SFRD contrast around the ring is strongest in the $\mathrm{H} \alpha+24 \mu \mathrm{m}$ map. The SFRD values found for $\mathrm{H} \alpha+24 \mu \mathrm{m}$ were checked against previous results by Wong et al. (2004). Wong et al. (2004) found a SFRD for the circumnuclear ring, based on $\mathrm{H} \alpha$ fluxes alone, of $0.04 M_{\odot} / \mathrm{yr} / \mathrm{kpc}^{2}$. We match this value with our $\mathrm{H} \alpha$ observations.

Comparing with the gas maps shown in Fig. 3, the SFR distribution is most similar to that of the atomic gas. This is surprising, given the fact that stars form from molecular not atomic gas. We speculate here that the young stars in the ring of NGC 4736 are energetic enough to dissociate large quantities of molecular gas in their immediate vicinity (without ionizing that gas as well), and will investigate this matter in an upcoming paper (Van der Laan, in prep.)

The star formation regions near the inflow points each cover about a fifth of the ring. The orbital time for a star at this radius $(r=0.9 \mathrm{kpc})$ is about $25 \mathrm{Myr}$, given a $220 \mathrm{~km} \mathrm{~s}^{-1}$ rotational velocity (value derived from the BIMA SONG first moment map). Therefore the duration of the starburst cannot be more than 5 Myr. This is also a reasonable time span to explain the difference between the $\mathrm{H} \alpha+24 \mu \mathrm{m}$, and NUV $+24 \mu \mathrm{m}$ and $\mathrm{FUV}+24 \mu \mathrm{m}$ maps. When we "subtract" the $\mathrm{H} \alpha$ distribution from either the NUV or FUV distribution (not shown), what remains is a more uniform distribution of flux, expected if the UV emission drops more slowly with time ( 5.5 Myr for $\mathrm{H} \alpha$, 11 Myr for NUV/FUV, as based on Starburst99 stellar burst synthesis models). The UV tracers are still tracing the previous "event" when they have moved through half the ring.

With these observations it is not possible to directly distinguish an age-gradient of star formation in the hot spots seen at the inflow points. Inspection of the observations shows that the hot spots are strongest in the $24 \mu \mathrm{m}$ observations and nearly unseen in the FUV and $\mathrm{H} \alpha$, implying they are (strongly) extincted regions. However, an equivalent width map of the $\mathrm{H} \alpha$ emission, given that a suitable continuum image is found, could potentially show age-related variations between the hot spots.

Averaged over the ring, the SFRD values correspond to a star formation rate density of $\sim 0.065 M_{\odot} / \mathrm{yr} / \mathrm{kpc}^{2}$. Within individual hotspots, the SFRD reaches more than $0.25 M_{\odot} / \mathrm{yr} / \mathrm{kpc}^{2}$. High SFRD are a common characteristic of circumnuclear star forming rings (Kormendy \& Kennicutt 2004; Kennicutt \& Evans 2012). Integrating over the full area of the ring leads to SFR values of $0.16,0.27$, and $0.25 M_{\odot} / \mathrm{yr}$, for $\mathrm{H} \alpha+24 \mu \mathrm{m}, \mathrm{NUV}+24 \mu \mathrm{m}$, and FUV $+24 \mu \mathrm{m}$, respectively. These values are lower than previously derived SFRs for the whole galaxy of $0.38 M_{\odot} / \mathrm{yr}$ (Calzetti et al. 2010), $0.43 M_{\odot} / \mathrm{yr}$ (Lee 2006), and $0.70 M_{\odot} / \mathrm{yr}$ (Skibba et al. 2011), but show the major role the circumnuclear ring plays in the overall star formation in NGC 4736.

\section{Radiation field in the ring}

In order to investigate the effect the young stars have on the local ISM, it is important to measure the emission from the dense neutral regions (the PDRs) close to the forming stars.

As a first step, it is important to estimate, and then remove, the contribution of [CII] from the diffuse, ionized ISM. Neutral Carbon has an ionization potential $(11.3 \mathrm{eV})$ which is slightly lower than that of Hydrogen $(13.6 \mathrm{eV})$. Consequently, it will be present in both the neutral and ionized ISM. The contribution of [CII] from the diffuse ionized ISM can be determined from an observed $[\mathrm{CII}] /[\mathrm{NII}] 205 \mu \mathrm{m}$ ratio, since $\mathrm{N}^{+}$emission arrises exclusively from the diffuse ionized medium (Bennett et al. 1994), 


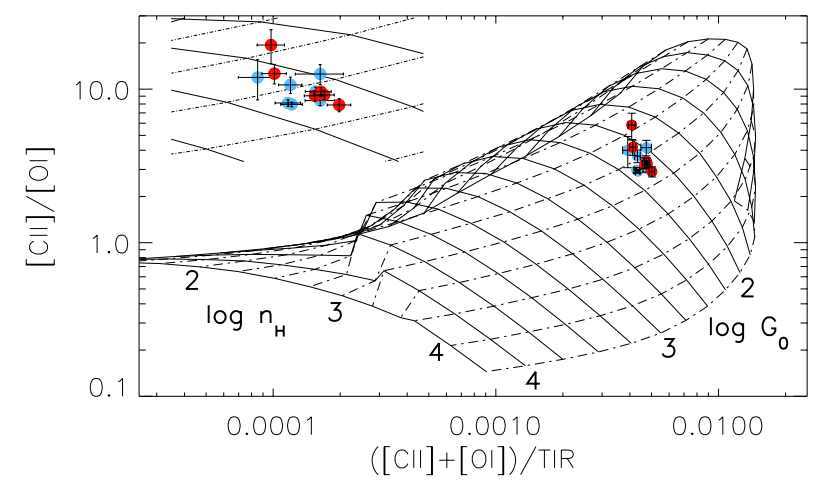

Fig. 5. $[\mathrm{CII}] /[\mathrm{OI}]$ vs. $([\mathrm{CII}]+[\mathrm{OI}]) / \mathrm{TIR}$ to determine the ambient UV radiation field incident on the PDR regions. The [CII] emission has been corrected for a $10 \%$ contribution from the diffuse ionized ISM. The underlying grid is the Kaufman et al. (1999) PDR model for different densities and stellar radiation field strengths. The inset sub-panel is a zoom-in of the region of interest. Data points in the east/west side of the ring are indicated in blue/red.

and the critical density of [CII] and [NII] $205 \mu \mathrm{m}$ are nearly the same. The observed $[\mathrm{CII}] /[\mathrm{NII}]$ ratio is nearly constant $(\sim 4)$ in the ionized ISM over a large range of electron density, $n_{\mathrm{e}}$. The difference between the predicted and measured $[\mathrm{CII}] /[\mathrm{NII}] 205 \mu \mathrm{m}$ ratio can then be used to estimate the ionized and neutral fractions of [CII] emission.

Since the $[\mathrm{NII}] 205 \mu \mathrm{m}$ line is not significantly detected in the NGC 4736 ring, we follow an analysis similar to the one discussed in Croxall et al. (2012) and use the $[\mathrm{CII}] /[\mathrm{NII}] 122 \mu \mathrm{m}$ ratio to determine the ionized $[\mathrm{CII}]$ emission. The $[\mathrm{CII}] /[\mathrm{NII}] 122 \mu \mathrm{m}$ ratio is dependent on electron density in the range $1-100 \mathrm{~cm}^{-3}$. While the [SIII] $18.7 / 33.5 \mu \mathrm{m}$ ratio is available from our IRS maps, the ratio [SIII]18.7/33.5 is sensitive to much higher electron densities in the range $10^{2-5} \mathrm{~cm}^{-3}$, and the $[\mathrm{SIII}]$ ratio $(0.56)$ is consistent with gas in the low density limit.

The [NII]122/205 ratio is sensitive to electron densities of $0.1-100 \mathrm{~cm}^{-3}$. Since the [NII] $205 \mu \mathrm{m}$ line is not detected in the ring, the observed [NII] $122 \mu \mathrm{m}$ signal-to-noise ratio in the ring $(5-10)$ can be used as a proxy for the upper limit of the [NII]122/205 ratio. From Beirão et al. (2012) we know that $[\mathrm{NII}] 122 \mu \mathrm{m} /[\mathrm{NII}] 205 \mu \mathrm{m}$ increases with increasing electron density, with the ratio reaching 10 at $n_{\mathrm{e}}=100 \mathrm{~cm}^{-3}$. Thus, in the ring we are dealing with an $n_{\mathrm{e}}$ lower than $100 \mathrm{~cm}^{-3}$, consistent with our Spitzer/IRS measurements. At an $n_{\mathrm{e}}$ of $100 \mathrm{~cm}^{-3}$ the $[\mathrm{CII}] /[\mathrm{NII}] 122 \mu \mathrm{m}$ ratio from the diffuse ionized component of the ISM would be 1.24 (this is an upper limit). All additional [CII] emission should be from the neutral component. In the region of the ring for which we have [NII] $122 \mu \mathrm{m}$ emission maps, we find $[\mathrm{CII}] /[\mathrm{NII}] 122 \mu \mathrm{m}$ ratios of $8-16$, with the value increasing away from the highest intensity regions. These values correspond to 85 to $92 \%$ contributions of the neutral phase to the [CII] emission. At lower electron density the [CII] fraction in the neutral phase rises. We therefore assume that $90 \%$ of the measured [CII] emission in the ring is from the neutral phase.

In Fig. 5 the (diffuse ionized emission corrected) $[\mathrm{CII}] /[\mathrm{OI}]$ and $([\mathrm{CII}]+[\mathrm{OI}]) /$ TIR ratios are overlaid on a PDR model from Kaufman et al. (1999, hereafter K99). As described in Sect. 2.1, the $[\mathrm{CII}],[\mathrm{OI}]$, and TIR fluxes were extracted in square apertures of $12^{\prime \prime}$ by $12^{\prime \prime}$ size, covering the circumnuclear ring. No difference is seen between the two (Fig. 5, red/blue points) sides of the ring. Based on these $[\mathrm{CII}] /[\mathrm{OI}]$ and $([\mathrm{CII}]+[\mathrm{OI}]) / \mathrm{TIR}$ ratios

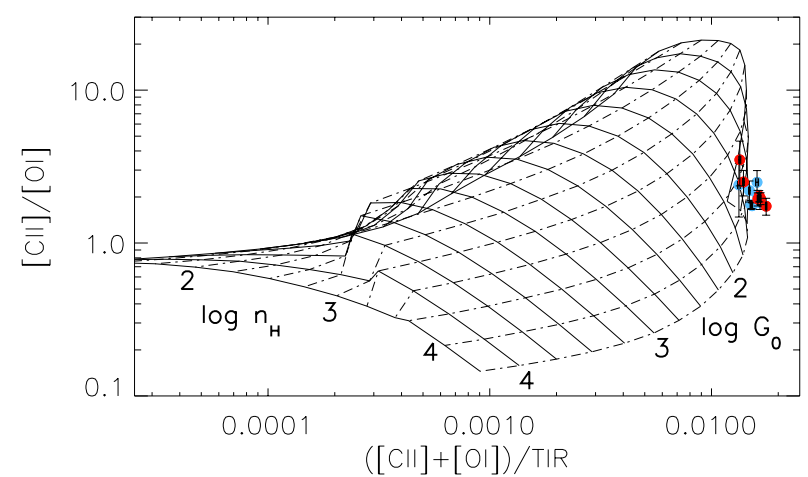

Fig. 6. Same as Fig. 5, except with a further assumption of $20 \%$ TIR, and $60 \%$ [CII] contribution from PDRs illuminated by a high radiation field intensity, with the rest originating from more diffuse neutral ISM components.

the radiation field $\mathrm{G}_{0}$, a dimensionless variable in multiples of the radiation field in the Solar neighborhood, in the PDR regions of the circumnuclear ring is found to have a uniform value of $\sim 200$, and the gas density, $n_{\mathrm{H}}$, in the PDR regions to be $\sim 10^{3} \mathrm{~cm}^{-1}$.

We can compare our $\mathrm{G}_{0}$ derived above from the FIR emission lines with the average $\langle\mathrm{U}\rangle$ obtained from modeling the dust emission. $\langle\mathrm{U}\rangle$ is a measure of the interstellar radiation field in units of the solar neighborhood (Draine \& Li 2007). For NGC 4736, $\langle\mathrm{U}\rangle$ is roughly 10 (Aniano et al., priv. comm.), about a factor of 20 lower than the $\mathrm{G}_{0}$ inferred above. This large mismatch between the radiation field intensity derived from the gas and dust has been noted in other studies as well (Croxall et al. 2012), and can be understood by noting that the PDR fraction, the fraction of the dust illuminated by the high intensity radiation field, can be much less than unity. Aniano et al. find a $f_{\mathrm{PDR}}$ (the fraction of dust mass illuminated by $\mathrm{G}_{0}$ greater than $\sim 110$ ) in the ring of order $20 \%$. If we apply this fraction to the TIR, and assume only $60 \%$ of the neutral [CII] emission is from dense PDRs illuminated by the high radiation field intensity, we can reconcile the two estimates (see Fig. 6).

\section{Heating-cooling balance}

In the "pearls-on-a-string" model, the heating in the ring as a function of azimuth is greatest close to the inflow points, followed by a slow decay as the stars age and the ring rotates. To see if the observed cooling in the ring follows this pattern, we compare the dust continuum flux and the far-IR line emission, against the expected available stellar heating.

\subsection{Energy input into the ISM}

By making several reasonable assumptions, the available energy for dust and gas heating from the newly formed stars in the ring can be derived from the measured SFRD. An instantaneous starburst was modeled using Starburst99 (Leitherer et al. 1999, 2010), assuming a standard Kroupa initial mass function and solar metallicity. The output synthetic line spectrum was integrated between $912 \AA$ and $1120 \AA(6-13.6 \mathrm{eV})$, to obtain the photoelectric heating energy as a function of time. The resulting available heating power per $M_{\odot}$ is shown in Fig. 7. As can be seen, the power in the $6 \mathrm{eV}$ to $13.6 \mathrm{eV}$ range of the integrated stellar population spectrum is strongly dependent on time. The flux plateaus 
T. P. R. van der Laan et al.: Heating and cooling of the neutral ISM in the NGC 4736 circumnuclear ring

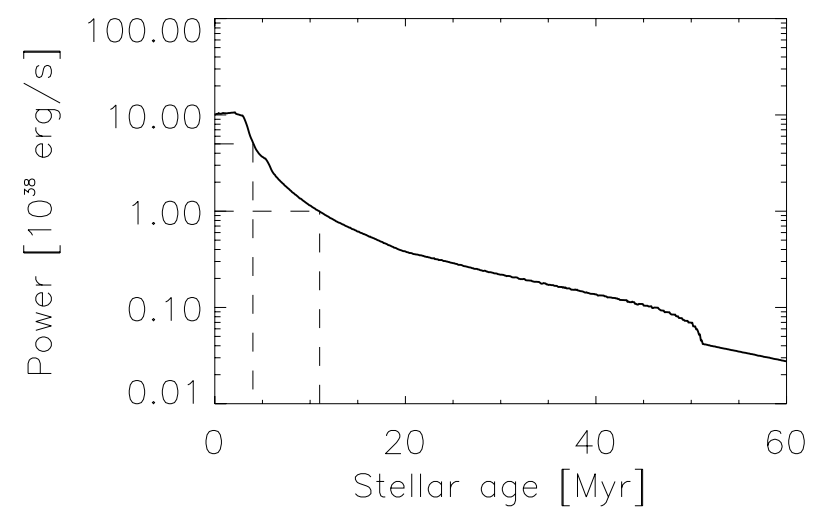

Fig. 7. Integrated FUV flux between $6-13.6 \mathrm{eV}$ in the case of an instantaneous starburst, rescaled to $1 M_{\odot}$. Within 4 Myr the energy has halved, and within $11 \mathrm{Myr}$ the energy has dropped by an order of magnitude. (Based on Starburst99 v6.0.3. models)

in the first $3 \mathrm{Myr}$, but within $11 \mathrm{Myr}$, it has dropped by an order of magnitude.

Instead of a single instantaneous starburst, physically the star formation will occur over a longer time interval. Like a Ferris wheel, the ring can be thought of as having discrete volume elements ("cabins"), scooping up gas over a fixed time interval as it enters onto the ring. In this model, each volume element will have a star formation event lasting a fixed fraction of the rotation time, with the mean age of the event depending on the distance from the inflow point. By tracing back the observed star formation under this premise, we can recreate the heating flux throughout the ring. As a reminder: the average SFRD measured in the ring was $0.065 M_{\odot} / \mathrm{yr} / \mathrm{kpc}^{2}$. To be able to compare the available heating to the observed TIR emission later on, we multiply by an average $24 \mu \mathrm{m} / \mathrm{UV}$ flux ratio of 0.33 , based on our observations, as a proxy of how much FUV flux is heating the dust, rather than immediately escaping the galaxy. This ratio is close to the measured dust/stellar flux ratio of 0.25 by Skibba et al. (2011).

\subsection{Comparison to dust}

A SFR history (for one full $25 \mathrm{Myr}$ orbit) is postulated where there are two starbursts of 5 Myr duration, 12.5 Myrs apart, with a burst SFR intensity of $0.01 M_{\odot} / \mathrm{yr}$ (thus a total of $5 \times 10^{4} M_{\odot}$ stellar mass per starburst is created). The heating curve is modeled by convolving the instantaneous starburst, shown in Fig. 7, with this proposed (box-)function of SFR history to obtain the instantaneous starburst heating as a function of time/position throughout the ring (left panel Fig. 8, grey saw-tooth curve). This heating curve is compared with the TIR continuum sampled throughout the ring (colored points). We may assume an instantaneous response from the dust to the heating, since the timescales here are of order $\sim \mathrm{Myr}$, while dust responds to heating on order $10^{4} \mathrm{yr}$, regardless of dust geometry (Draine, priv. comm.). In Fig. 8 the correspondence between the measured TIR continuum and the modeled heating is very good. However, it is clear that the heating power falls too steeply around the ring, and that the second peak is significantly lower than the first. A much better match (black curve) can be made by shortening both initial bursts, lowering of the second burst to $0.008 M_{\odot} / \mathrm{yr}$, and adding two short bursts of star formation at $\sim 8.75-10 \mathrm{Myr}$ and 20-21.25 Myr. The inclusion of the latter is a divergence from the "pearls-on-a-string" scenario.

\subsection{Comparison to PDR gas}

To compare the response of the PDR gas to the modeled heating we need an observational measure of the photoelectric heating efficiency. This is the ratio of photon energy going into gas heating to the total available energy. This ratio, typically taken as $([\mathrm{CII}]+[\mathrm{OI}]) / \mathrm{TIR}$, is equal to the ratio of gas cooling line flux to TIR continuum flux. In Fig. 9 this gas to dust ratio is plotted against, the ratio $\left\langle v S_{v}\right\rangle_{70} /\left\langle v S_{v}\right\rangle_{100}$, which is used as a proxy for the dust temperature. From Fig. 9 it can be seen that the photoelectric heating efficiency shows very little spread over the circumnuclear ring, with values of $0.004-0.006$, about $0.5 \%$.

In the right panel of Fig. 8, this fixed $0.5 \%$ scaling has been applied to the $[\mathrm{CII}]+[\mathrm{OI}]$ flux in each region. It is immediately clear that the spread in the emission line flux is due to the FUV heating variation. The two cooling lines again follow the modeled FUV heating quite closely, although we lack the data points away from the inflow points to confirm the secondary star forming events, due to limited Herschel spectral coverage.

The photoelectric heating efficiencies are comparable to the values seen for other kpc-sized star forming regions, as for example in Croxall et al. (2012) or Parkin et al. (2013). These studies find values of $\sim 0.5 \%$ at lower dust temperatures, with a significant drop of heating efficiency at higher dust temperatures. However, Lebouteiller et al. (2012) and Parkin et al. (2014) do not find a reduction in the gas heating efficiency at higher dust temperatures in a star forming region of the LMC, and the disk of Centaurus A, respectively. The NGC 4736 ring does, however, have a higher photoelectric heating efficiency, at only marginally higher dust temperature, than starburst ring in NGC 1097 ring (Beirão et al. 2012).

\section{Implications for ring star formation theory}

We started this investigation to study if, following the "pearlson-a-string" paradigm for circumnuclear star forming rings, all star formation takes place at the inflow points of the ring. In this scenario, as the system rotates, the subsequent star forming events should create a clear azimuthal age gradient (Böker et al. 2008).

Our simple modeling of the PDR dust and gas heating as function of azimuth requires secondary star formation events to be consistent with the observed cooling flux. While the exact strength, timing, and duration of these secondary events in the NGC 4736 ring is model dependent, it is clear that their absence leads to a too large contrast in heating/cooling flux around the ring. Since the FUV heating power available from young stars diminishes by an order of magnitude in $11 \mathrm{Myr}$, it seems unlikely that the drop be compensated for by stellar "after-glow".

Are these "secondary" star forming events truly random, taking place from left-over gas from the "primary" event? Or, are both events part of the "popcorn" scenario (Elmegreen 1994), where star formation occurs anywhere in the ring? Given the relative strength of the events at both inflow points we consider this unlikely. This seems consistent with the fact that a number of galaxies show strong star formation events at the inflow points of their circumnuclear rings (Ryder et al. 2001; Allard et al. 2005; Böker et al. 2008; Mazzuca et al. 2008; Van der Laan et al. 2013a,b).

A combination of "pearls-on-a-string" and "popcorn" seems like a reasonable scenario to explain the star formation in the ring of NGC 4736. We know star formation is not a $100 \%$ efficient process. Star formation efficiencies (SFE) are usually estimated to be of order a few percent. Although there may be an indication 

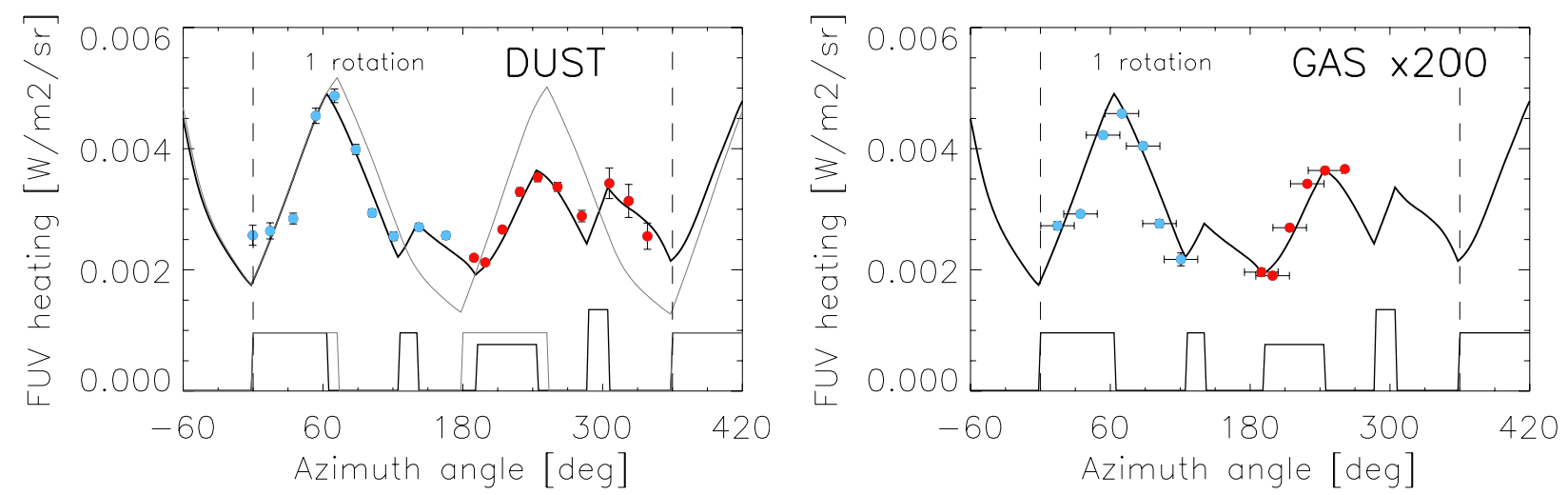

Fig. 8. Left: available FUV heating flux (black saw-tooth line) as a function of azimuth, derived from an proposed star formation history. The proposed star formation history is indicated at the bottom of the panel (black box function). The grey lines (underlying the black) are an alternative example of a star formation history and FUV heating flux. The observed FIR (dust) flux is shown in blue/red (east/west side of the ring). Details in the text. Right: similar, except now for the $[\mathrm{CII}]+[\mathrm{OI}]$ (gas) emission lines. The FoV of the Herschel spectra does not cover the full ring, thus for several apertures the gas information is lacking.

that the SFE in the center of NGC 4736 is actually higher (10\%, Sandstrom, priv. comm.), even then a significant portion of gas remains. Therefore, we can ask if the remaining gas has enough chance to cool and is dense enough to collapse again.

The total gas mass surface density in the ring remains $\sim 50-80 M_{\odot} / \mathrm{pc}^{2}$ away from the inflow points (Fig. 3). This is higher than the $\sim 8 M_{\odot} / \mathrm{pc}^{2}$ star formation threshold noticed in Bigiel et al. (2008). Additionally, while the temporal distance between the inflow points and these modeled secondary bursts is short (a few Myr), it is comparable to free fall times of giant molecular clouds.

Can we expect this hybrid star formation scenario in all circumnuclear rings? The life times of circumnuclear rings are of order $1-2$ Gyr. Inflow rates of gas onto the ring of $2 M_{\odot} / y r$ are a reasonable upper limit (Van der Laan et al. 2011, 2013a), although values of $0.1-0.25 M_{\odot} / y r$ are more likely. So, even at extreme SFEs and low gas inflow rates, the gas mass in a ring will see an increase of about $10^{8} M_{\odot}\left(0.1 M_{\odot} / \mathrm{yr} \times 1 \mathrm{Gyr}\right)$ over the life time of the ring. Given the observed range of radii and widths found for circumnuclear rings $(40-1000 \mathrm{pc}$ radii, $\sim r / 2$ widths, Comerón et al. 2010), this translates easily to gas mass surface densities similar to those found in NGC $4736\left(10-10^{2} M_{\odot} / \mathrm{pc}^{2}\right)$. Thus, the prevalence of "popcorn" star formation events should increase with the lifetime of the circumnuclear ring, even if starbursts, "pearls-on-a-string", continue to be at the inflow points.

\section{Summary}

New Herschel FIR observations of the circumnuclear ring in NGC 4736 have been combined with archival observations of the star formation, and atomic and molecular gas distributions. This combination of data sets is used to characterize the mode of star formation in the ring. Due to the circular orbits of the ring itself, gas and dust will only experience large-scale shocks and compression at the inflow points, the locations where material transitions onto the ring. Star formation events, therefore, should be uniquely found close to the inflow points. We have investigated the heating and cooling of the interstellar medium in the ring of NGC 4736, under this assumption. The stellar FUV flux available for heating was derived from measurements of the star formation rate, geometry-based estimates of time scales, and standard assumptions on the initial mass function. The FIR continuum and gas emission line fluxes were measured from Herschel/PACS and archival Spitzer observations, and used to

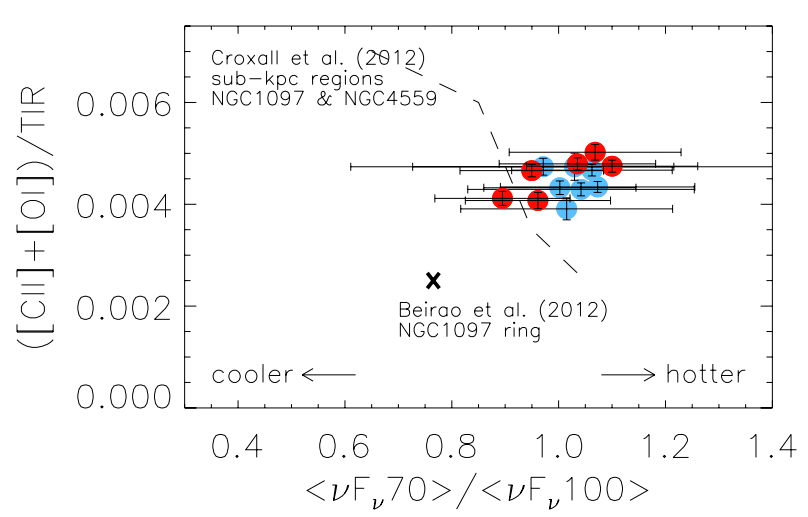

Fig. 9. Gas heating efficiency proxy, $([\mathrm{CII}]+[\mathrm{OI}]) / \mathrm{TIR}$, as a function of the dust temperature (blue (east) and red (west) dots). For comparison, the results from Beirão et al. (2012) in the circumnuclear star forming ring of NGC 1097 ("x"), and Croxall et al. (2012) in sub-kpc regions in NGC 1097 and NGC 4559 (dashed line) are indicated. The [CII] has been corrected for its $10 \%$ diffuse ionized component.

estimate the heating and cooling of the dust and gas around the ring.

We find that, while heating from a significant burst of star formation at the inflow points can explain the gas and dust cooling there, an additional star formation component is required at other positions in the ring. This additional component is smaller than the star formation events at the inflow points, and most likely arises from the general increase of gas density in the ring over its lifetime. Therefore, in the NGC 4736 ring, it is a combination of the "popcorn" and "pearls-on-a-string" models that best explains the data.

Acknowledgements. This project was started during a "11/"12 IPAC visiting graduate fellowship awarded to T.v.d.L. T.v.d.L. would like to thank IPAC, especially P. Appleton and L. Armus, for hosting her.

\section{References}

Allard, E. L., Peletier, R. F., \& Knapen, J. H. 2005, ApJ, 633, L25 Aniano, G., Draine, B. T., Gordon, K. D., \& Sandstrom, K. 2011, PASP, 123, 1218

Aniano, G., Draine, B. T., Calzetti, D., et al. 2012, ApJ, 756, 138

Bakes, E. L. O., \& Tielens, A. G. G. M. 1994, ApJ, 427, 822

Beirão, P., Armus, L., Helou, G., et al. 2012, ApJ, 751, 144

Bennett, C. L., Fixsen, D. J., Hinshaw, G., et al. 1994, ApJ, 434, 587 
T. P. R. van der Laan et al.: Heating and cooling of the neutral ISM in the NGC 4736 circumnuclear ring

Bigiel, F. Leroy, A., Walter, F, et al. 2008, AJ, 136, 2846

Böker, T., Falcón-Barroso, J., Schinnerer, E., Knapen, J. H., \& Ryder, S. 2008, AJ, 135, 479

Bresolin, F., \& Kennicutt, Jr., R. C. 1997, AJ, 113, 975

Calzetti, D., Wu, S.-Y., Hong, S., et al. 2010, ApJ, 714, 1256

Comerón, S., Knapen, J. H., Beckman, J. E., et al. 2010, MNRAS, 402, 2462

Contopoulos, G., \& Papayannopoulos, T. 1980, A\&A, 92, 33

Croxall, K. V., Smith, J. D., Wolfire, M. G., et al. 2012, ApJ, 747, 81

Dale, D. A., Aniano, G., Engelbracht, C. W., et al. 2012, ApJ, 745, 95

Draine, B. T., \& Li, A. 2007, ApJ, 657, 810

Elmegreen, B. G. 1994, ApJ, 425, L73

Falcón-Barroso, J., Ramos Almeida, C., Böker, T., et al. 2014, MNRAS, 438, 329

González Delgado, R. M., Leitherer, C., \& Heckman, T. M. 1999, ApJS, 125, 489

Grouchy, R. D., Buta, R. J., Salo, H., \& Laurikainen, E. 2010, AJ, 139, 2465

Hao, C.-N., Kennicutt, R. C., Johnson, B. D., et al. 2011, ApJ, 741, 124

Helfer, T. T., Thornley, M. D., Regan, M. W., et al. 2003, ApJS, 145, 259

Helou, G., Malhotra, S., Hollenbach, D. J., Dale, D. A., \& Contursi, A. 2001, ApJ, 548, L73

Kaufman, M. J., Wolfire, M. G., Hollenbach, D. J., \& Luhman, M. L. 1999, ApJ, 527,795

Kennicutt, R. C., \& Evans, N. J. 2012, ARA\&A, 50, 531

Kennicutt, Jr., R. C., Armus, L., Bendo, G., et al. 2003, PASP, 115, 928

Kennicutt, R. C., Calzetti, D., Aniano, G., et al. 2011, PASP, 123, 1347

Knapen, J. H., Stedman, S., Bramich, D. M., Folkes, S. L., \& Bradley, T. R. 2004, A\&A, 426, 1135
Kormendy, J., \& Kennicutt, Jr., R. C. 2004, ARA\&A, 42, 603

Lebouteiller, V., Cormier, D., Madden, S. C., et al. 2012, A\&A, 548, A91

Lee, J. C. 2006, Ph.D. Thesis, Proquest Dissertations And Theses 2006, Arizona: The University of Arizona, United States

Leitherer, C., \& Heckman, T. M. 1995, ApJS, 96, 9

Leitherer, C., Schaerer, D., Goldader, J. D., et al. 1999, ApJS, 123, 3

Leitherer, C., Ortiz Otálvaro, P. A., Bresolin, F., et al. 2010, ApJS, 189, 309

Mazzuca, L. M., Knapen, J. H., Veilleux, S., \& Regan, M. W. 2008, ApJS, 174, 337

Murphy, E. J., Condon, J. J., Schinnerer, E., et al. 2011, ApJ, 737, 67

Parkin, T. J., Wilson, C. D., Schirm, M. R. P., et al. 2013, ApJ, 776, 65

Parkin, T. J., Wilson, C. D., Schirm, M. R. P., et al. 2014, ApJ, 787, 16

Poglitsch, A., Waelkens, C., Geis, N., et al. 2010, A\&A, 518, L2

Regan, M. W., \& Teuben, P. 2003, ApJ, 582, 723

Roussel, H. 2013, PASP, 125, 1126

Ryder, S. D., Knapen, J. H., \& Takamiya, M. 2001, MNRAS, 323, 663

Sandstrom, K. M., Leroy, A. K., Walter, F., et al. 2013, ApJ, 777, 5

Skibba, R. A., Engelbracht, C. W., Dale, D., et al. 2011, ApJ, 738, 89

Smith, J. D. T., Draine, B. T., Dale, D. A., et al. 2007, ApJ, 656, 770

Van der Laan, T. P. R., Schinnerer, E., Boone, F., et al. 2011, A\&A, 529, A45

Van der Laan, T. P. R., Schinnerer, E., Emsellem, E., et al. 2013a, A\&A, 551, A81

Van der Laan, T. P. R., Schinnerer, E., Emsellem, E., et al. 2013b, A\&A, 556, A98

Walter, F., Brinks, E., de Blok, W. J. G., et al. 2008, AJ, 136, 2563

Weingartner, J. C., \& Draine, B. T. 2001, ApJS, 134, 263

Wong, T., Blitz, L., \& Bosma, A. 2004, ApJ, 605, 183 